\title{
Trends in Outcomes of Patients With Metastatic Cancer Undergoing Intubation and Mechanical Ventilation: Results of the National Hospital Discharge Survey
}

\author{
Arjun Gupta, MDa; Avash Das, MBBS ${ }^{\mathrm{b}}$ Raseen Tariq, MD; Nizar Bhulani, MBBS, MPH ${ }^{\mathrm{a}, \mathrm{d}}$; \\ Naveen Premnath, MD; ; Dipesh Solanky, BSc; Ryan D. Frank, MSf; David Johnson, MDa; \\ Sahil Khanna, MD, MSc; and Muhammad S. Beg, MDd,
}

\begin{abstract}
Background: There has been an overall decline in intensive care unit mortality over the past 2 decades, including in patients undergoing intubation and mechanical ventilation (MV). Whether this decline extends to patients with metastatic cancer remains unknown. We analyzed the outcomes of patients with metastatic cancer undergoing intubation/MV using the National Hospital Discharge Survey (NHDS) database from 2001 to 2010. Methods: Diagnosis and procedure codes were used to identify patients with metastatic cancer who underwent intubation/MV. Demographics, diagnoses, length of stay (LOS), and discharge information were abstracted. Multivariate linear and logistic regression models with weighted analysis were conducted to study trends in outcomes. Results: During the 10-year study period, 200,350 patients with metastatic cancer and who underwent intubation/MV were identified; the mean age was 65.3 years and $46.2 \%$ were men. There was an increase in the total number of patients with metastatic cancer who underwent intubation/MV during the study period, from $36,881$ in $2001-2002$ to 51,003 in 2009-2010 ( $P<.001)$. The overall inpatient mortality rate was $57.3 \%$, discharge to a care facility (DTCF) rate was $40.9 \%$ among patients alive at discharge, and mean LOS was 11.1 days. No significant trends were seen in rates of mortality, DTCF, or LOS from 2001 to 2010. Conclusions: In this national database, there was an increase in the number of patients with metastatic cancer who underwent intubation/MV. These patients had high rates of inpatient mortality and DTCF, which did not improve during the study period. Therefore, novel solutions are required to improve outcomes for these patients.
\end{abstract}

J Natl Compr Canc Netw 2018;16(3):286-292 doi: 10.6004 ljnccn.2017.7053

Advancements in the treatment of cancer, including metastatic cancer, over the past few decades have led to improved survival rates. ${ }^{1}$ Consequently, an increasing number of patients with advanced cancer are eligible for aggressive intensive care unit (ICU)-level care, including intubation and mechanical ventilation (MV). ${ }^{2}$ Traditionally, the prognosis of patients with metastatic cancer requiring intensive care was poor, ${ }^{3}$ and the role of aggressive interventions in this setting was questionable.

From the aDepartment of Internal Medicine, University of Texas Southwestern Medical Center, Dallas, Texas; 'bivision of Cardiology, Massachusetts General Hospital, Boston, Massachusetts; 'Division of Gastroenterology and Hepatology, Mayo Clinic, Rochester, Minnesota; 'Division of Oncology, University of Texas Southwestern Medical Center, Dallas, Texas; elcahn School of Medicine at Mount Sinai St Luke's-Roosevelt Hospital Centre, New York, New York; and fDivision of Biomedical Statistics and Informatics, Mayo Clinic, Rochester, Minnesota.

Submitted July 27, 2017; accepted for publication October 26, 2017.

The authors have disclosed that they have no financial interests,
Within the past few decades, ICU outcomes have improved drastically, owing to better management techniques, early identification of sicker patients, and more lenient admission criteria for ICUs. One recent study reported a $35 \%$ relative decrease in the mortality of patients admitted to the ICU from 1988 to 2012, despite an increase in age and severity of illness, as well as an increase in discharges to care facilities. ${ }^{4}$ On stratified analysis, this improvement in mortality was similar among patients

arrangements, affiliations, or commercial interests with the manufacturers of any products discussed in this article or their competitors.

Previously presented in abstract form at the 2017 Palliative and Supportive Care in Oncology Symposium; October 23, 2017; San Diego, California (J Clin Oncol 2017;35[Suppl]:Abstract 69).

Correspondence: Muhammad S. Beg, MD, Division of Hematology/ Oncology, Department of Internal Medicine, University of Texas Southwestern Medical Center, 5323 Harry Hines Boulevard, Dallas, TX 75390-8852. E-mail: Muhammad.Beg@UTSouthwestern.edu 
who did and did not receive MV. The integration of early palliative care in suitable patients with cancer has allowed for improved identification of those who could potentially benefit from ICU care. ${ }^{5}$ Moreover, early palliative care can lead to improved patient and caregiver outcomes and reduced futile ICU use. Accordingly, ASCO recommends integrating dedicated palliative care services into the care of patients with advanced cancer early in the disease course, concurrent with active treatment. ${ }^{6}$

Despite these interventions, a review of Medicare data suggests that the proportion of patients with cancer admitted to ICUs in the last month of life increased from $23.7 \%$ in the mid-2000s to $30.2 \%$ in $2012 .{ }^{7}$ Outcomes for these patients are poor, with one cohort demonstrating an inpatient mortality rate of $54 \%$ for those with metastatic cancer undergoing intubation/MV. ${ }^{8} \mathrm{~A}$ meta-analysis of 42 studies showed that only $5.6 \%$ of patients with metastatic cancer undergoing in-hospital cardiopulmonary resuscitation survived to discharge, ${ }^{9}$ although a trend was seen toward improving survival over time. Whether outcomes of patients with metastatic cancer admitted to the ICU and requiring intubation/ MV have mirrored the overall decline in ICU-related mortality has not been studied. Limited ICU bed availability, concerns about the inappropriate use of limited resources, and uncertainties regarding outcome make ICU admission decisions for critically ill patients with cancer a challenging issue. Therefore, we used the National Hospital Discharge Survey (NHDS) database to evaluate trends in the outcomes of hospitalized patients with metastatic cancer undergoing intubation/MV from 2001 to 2010.

\section{Methods}

\section{Data Source}

The NHDS is a national survey, conducted annually since 1965, that collects discharge information from nonfederal short-stay hospitals (where the average length of stay [LOS] is $<30$ days). Federal, military, and Department of Veterans Affairs hospitals, as well as hospital units of institutions (eg, prison hospitals) and hospitals with fewer than 6 beds staffed for patient use, are excluded. NHDS collected data from a sample of approximately 500 hospitals from 2000 to 2007, and from 239 hospitals from 2008 to 2010. The survey uses a stratified multistage cluster sampling de- sign, and the unit of analysis is the hospital discharge. The NHDS has been used extensively to study trends in the incidence and outcomes of several conditions (https://www.cdc.gov/nchs/data/nhds/nhds_article_ list.pdf). Since 2010, the NHDS has been replaced by a new survey, the National Hospital Care Survey (NHCS), which integrates inpatient data with the emergency department, outpatient department, and ambulatory surgery center data. Available information includes patient diagnosis codes, demographics, type of admission (ie, emergent/urgent or elective), LOS, in-hospital mortality, and discharge location. The database is accessible online at http://www.cdc. gov/nchs/nhds.htm. All diagnoses are based on ICD9-CM codes.

\section{Data Collection}

Data collected and analyzed included age, sex, race, admission type (urgent/emergent vs elective), hospital characteristics, LOS, discharge location, and mortality for all patients discharged between January 1, 2001, and December 31, 2010. This period was chosen to identify a contemporary cohort of patients with metastatic cancer who were eligible to experience advancements in ICU management and more liberal ICU admission policies, and the earlier integration of palliative care in the latter period of the study. $\mathrm{Pa}$ tients aged $<18$ years were excluded from analyses.

\section{Definitions of Variables}

Patients with metastatic cancer were identified using ICD-9-CM diagnosis codes (supplemental eTable 1, available with this article at JNCCN.org). We included only metastatic solid cancers in this analysis because the clinical course of hematologic cancers is different. These ICD-9-CM codes for metastatic cancer have been described previously. ${ }^{8,10,11}$ Procedural codes were used to identify patients with intubation/MV $(96.70,96.71,96.72) .{ }^{8}$ The NHDS classifies discharges into one of several categories: routine and/or discharged home, discharged to a short-term healthcare facility, discharged to a longterm healthcare facility, left against medical advice, death during hospitalization, or unknown discharge status. Patients discharged to healthcare facilities (either short- or long-term) were classified as "discharged to a care facility" (DTCF). Patients with discharge disposition set to unknown/other discharge status were not included in the discharge analysis. 
Gupta et al

Comorbidities including congestive heart failure, valvular disease, pulmonary circulatory disease, peripheral vascular disease, diabetes mellitus with complications, hypertension, liver disease, and obesity were identified using ICD-9-CM diagnosis codes and diagnosis-related group codes. They were assessed using the Charlson comorbidity index. Patients with heart failure and stroke undergoing intubation/MV were also identified using ICD-9-CM codes (supplemental eTable 1), and their incidence and outcomes were also studied to compare and contrast with the trends in metastatic cancer. Patients in the "sepsis" cohort were identified to study whether the diagnoses varied over time. Utilization of palliative care was identified using ICD-9-CM code V66.7.

\section{Statistical Analyses}

Demographic and clinical data were summarized using frequencies and percentages for categorical variables, and means/medians for continuous variables. To identify any outliers, we repeated the analyses using a logarithmic transformation on LOS, with similar results. All analyses were performed using SAS 9.4 (SAS Institute, Cary, NC). Weighted analyses were used to obtain nationwide estimates and account for the stratified sampling process of the NHDS database.

$t$-Tests and chi-square tests were used to compare medians and proportions, respectively, in patients who died as inpatients or survived to discharge. Trends in DTCF and in-hospital mortality were analyzed using weighted logistic regression, and in LOS using linear regression. Multivariate models were used to additionally adjust for the effects of age, sex, race, admission type, hospital characteristics, primary cancer, and Charlson comorbidity index score. When appropriate, continuous variables were reported as means or medians. Categorical variables were reported as percentages and compared using odds ratios (ORs) and adjusted ORs (aORs), and 95\% confidence intervals (CIs). All analyses were performed using SAS 9.4, and all tests were 2 -sided. $P<.05$ was determined to be statistically significant.

\section{Results}

\section{Demographics and Baseline Data}

An estimated 200,350 adult hospital discharges with metastatic cancer underwent intubation/MV during the study period. An additional 17,531 patients who had disposition status as "alive, disposition not stated" or "not stated or reported" were excluded from the study. Table 1 presents the study patient demographics, hospital characteristics, and patient comorbidities. The mean age of participants was 65.3 years, with $53.6 \%$ aged $>65$ years. Overall, $77.2 \%$ of these were classified as emergent/urgent on admission. Ap-

\begin{tabular}{|c|c|c|c|c|}
\hline & Total, \%a & Alive, $\%^{a}$ & Died, \%a & $P$ Value $^{\mathrm{b}}$ \\
\hline Total, N & 200,350 & 85,559 & 114,791 & \\
\hline Mean age (SD), y & $65.3(12.5)$ & $66.5(12.3)$ & $64.4(12.6)$ & .06 \\
\hline \multicolumn{5}{|l|}{ Sex } \\
\hline Male & 46.2 & 49.9 & 43.4 & .14 \\
\hline \multicolumn{5}{|l|}{ Age distribution } \\
\hline $18-49 y$ & 10.8 & 10.2 & 11.2 & \multirow{3}{*}{.06} \\
\hline $50-65 y$ & 35.7 & 30.6 & 39.4 & \\
\hline$>65 y$ & 53.6 & 59.1 & 49.4 & \\
\hline \multicolumn{5}{|l|}{ Race } \\
\hline White & 64.8 & 64.8 & 64.7 & \multirow{5}{*}{.18} \\
\hline African American & 13.5 & 12.4 & 14.4 & \\
\hline Asian & 1.6 & 1.5 & 1.6 & \\
\hline Others & 3.2 & 1.6 & 4.4 & \\
\hline Not stated & 17.0 & 19.7 & 14.9 & \\
\hline \multicolumn{5}{|l|}{ Geographic region } \\
\hline Northeast & 28.3 & 26.8 & 29.4 & \multirow{4}{*}{.61} \\
\hline Midwest & 20.2 & 22.9 & 18.2 & \\
\hline South & 33.8 & 33.6 & 34.0 & \\
\hline West & 17.7 & 16.7 & 18.5 & \\
\hline \multicolumn{5}{|l|}{ Admission type } \\
\hline Emergent/Urgent & 77.2 & 71.1 & 81.8 & \multirow{3}{*}{$<.001$} \\
\hline Elective & 12.4 & 18.8 & 7.7 & \\
\hline Not stated & 10.4 & 10.1 & 10.5 & \\
\hline \multicolumn{5}{|c|}{ Principal expected source of payment } \\
\hline Medicare & 52.5 & 56.0 & 49.9 & \multirow{7}{*}{.31} \\
\hline Medicaid & 8.4 & 6.3 & 10.0 & \\
\hline Blue Cross/Blue Shield & 9.5 & 10.6 & 8.7 & \\
\hline HMO/PPO & 13.5 & 11.9 & 14.7 & \\
\hline Other private & 9.3 & 7.7 & 10.5 & \\
\hline Self-pay & 2.8 & 2.2 & 3.2 & \\
\hline Other/Not stated & 4.0 & 5.3 & 3.0 & \\
\hline \multicolumn{5}{|l|}{ Hospital ownership } \\
\hline Nonprofit & 78.0 & 79.3 & 77.0 & \multirow{3}{*}{.86} \\
\hline Proprietary & 9.4 & 9.1 & 9.7 & \\
\hline Government & 12.5 & 11.6 & 13.2 & \\
\hline \multicolumn{5}{|l|}{ Number of hospital beds } \\
\hline $6-99$ & 9.2 & 10.3 & 8.4 & \multirow{5}{*}{.26} \\
\hline $100-199$ & 19.5 & 19.0 & 19.9 & \\
\hline $200-299$ & 22.7 & 20.8 & 24.2 & \\
\hline $300-499$ & 29.2 & 26.4 & 31.4 & \\
\hline$>500$ & 19.3 & 23.6 & 16.2 & \\
\hline \multicolumn{5}{|l|}{ Primary cancer site } \\
\hline Lung & 1.7 & 1.0 & 2.3 & \multirow{6}{*}{.02} \\
\hline Breast & 19.1 & 22.3 & 16.8 & \\
\hline Colorectal & 4.6 & 6.7 & 3.0 & \\
\hline Genitourinary & 3.0 & 4.4 & 2.0 & \\
\hline Other or $>1$ cancer subtype & 48.3 & 42.1 & 52.8 & \\
\hline No primary identified & 23.2 & 23.6 & 22.9 & \\
\hline $\begin{array}{l}\text { Mean Charlson comorbidity } \\
\text { score (SD) }\end{array}$ & $8.0(1.4)$ & $8.1(1.3)$ & $7.8(1.4)$ & .01 \\
\hline \multicolumn{5}{|c|}{$\begin{array}{l}\text { all data represent proportion of cases unless otherwise stated. } \\
\text { b } P \text { value adjusted for age, sex, race, admission type, primary cancer, and Charlson } \\
\text { comorbidity score. There is only one } P \text { value per variable because it is testing to } \\
\text { see whether there is a difference in proportions between those who died versus } \\
\text { those who did not. The } P \text { value used for the categorical variables was a weighted } \\
\text { Rao-Scott chi-square test. For the continuous variables, weighted univariable linear } \\
\text { regression was used. }\end{array}$} \\
\hline
\end{tabular}


proximately one-third of the discharges (33.8\%) occurred in the geographic area marked as the "South." More than half (51.9\%) occurred in medium-sized hospitals (200-499 beds), and more than a quarter $(25.5 \%)$ occurred in the last 2 years of the study (Table 2). The most common comorbid diseases/ conditions included fluid and electrolyte disorders $(30.2 \%)$, chronic pulmonary disease $(23.4 \%)$, congestive heart failure (10.1\%), and anemia (7.7\%). Medicare was listed as the primary expected source of payment in $52.5 \%$ of discharges. From 2001 to 2010 , there was a significant increase in total number of patients, from 36,881 in 2001-2002 to 51,003 in 2009-2010 (Figure 1; $P$ value for trend <.001). The rates of intubation/MV among all patients with metastatic cancer increased from 2.2\% in 2001-2002 to $2.7 \%$ in $2009-2010(P=.03)$.

\section{Outcomes Data}

The overall inpatient mortality rate was $57.3 \%$, DTCF rate was $15.2 \%$ (40.9\% among patients alive at discharge), and mean LOS was 11.1 days. Further trends in outcomes are presented in Table 2. Outcomes varied across the study period, with the longest LOS (12.5 days) and highest number of DTCFs in 2005-2006. The rate of inpatient mortality was highest in the last 2 years of the study (2009-2010; $60.8 \%$ ). No significant trends in DTCF or mortality, nor in adjusted mean difference in LOS were observed over the 10-year study period (Figure 2).

\section{Use of Palliative Care}

An estimated 6,690 patients (3.3\%) received palliative care, whereas 193,660 (96.7\%) did not. There was a steady increase in the use of palliative care during the 10-year study period, from $0 \%$ in 2000 to $3.2 \%$ in 2009 , and an increase to $17.4 \%$ in 2010 ( $P$ value for trend $<.001$ ). Patients who received palliative care had higher inpatient mortality (89.7\%) compared with those who did not $(56.2 \%)$. On multivariable analysis, palliative care was significantly associated with inpatient mortality (aOR, 9.61; 95\% CI, 2.20-42.04; $P=.003$ ). There was no statistically significant difference in rates of DTCF and LOS between patients who did and did not receive palliative care in multivariable analysis.

\section{Data in Other Diagnoses and Trends in Sepsis}

An estimated 1,549,460 and 232,990 adult hospital discharges with heart failure and stroke, respectively, underwent intubation/MV during the study period; there was an increase in the numbers of these patients through the study period. The number of patients with heart failure who underwent intubation/MV increased from 280,820 in 20012002 to 367,420 in 2009-2010, whereas the number of patients with stroke who underwent intubation/ MV increased from 40,960 in 2001-2002 to 63,960 in 2009-2010. No significant trend was seen in the outcomes of these patients across the study period (supplemental eTable 2).

The percentage of patients with metastatic cancer who underwent intubation/MV who had a diagnosis of sepsis increased consistently throughout the study period, from $3.4 \%$ in $2001-2002$ to $8.3 \%$ in 2009-2010 (supplemental eFigure 1).

\section{Discussion}

From 2001 to 2010 in this large, national, hospitalized patient sample, the number of patients with metastatic cancer who required intubation/MV increased,

\begin{tabular}{|c|c|c|c|c|c|c|}
\hline Covariate & $\begin{array}{c}2001-2002 \\
n, \%\end{array}$ & $\begin{array}{c}2003-2004 \\
n, \%\end{array}$ & $\begin{array}{c}2005-2006 \\
n, \%\end{array}$ & $\begin{array}{c}2007-2008 \\
n, \%\end{array}$ & $\begin{array}{c}2009-2010 \\
n, \%\end{array}$ & $\begin{array}{c}\text { Overall } \\
\mathrm{n}, \%\end{array}$ \\
\hline Total, N & 36,881 & 31,775 & 41,264 & 39,427 & 51,003 & 200,350 \\
\hline Mean length of stay (SD), d & $10.8(10.7)$ & $10.2(9.9)$ & $12.5(12.0)$ & $11.9(12.4)$ & $10.0(10.5)$ & $11.1(11.2)$ \\
\hline \multicolumn{7}{|l|}{ Discharge to care facility ${ }^{a}$} \\
\hline No & $7,953(66.3 \%)$ & $6,483(58.7 \%)$ & $10,136(59.1 \%)$ & $6,337(51.6 \%)$ & $9,312(59.8 \%)$ & $40,221(59.1 \%)$ \\
\hline Yes & $4,042(33.7 \%)$ & $4,555(41.3 \%)$ & $7,011(40.9 \%)$ & $5,949(48.4 \%)$ & $6,250(40.2 \%)$ & $27,807(40.9 \%)$ \\
\hline \multicolumn{7}{|l|}{ Vital status } \\
\hline Alive & $15,242(41.3 \%)$ & $13,956(43.9 \%)$ & $19,943(48.3 \%)$ & $16,405(41.6 \%)$ & $20,013(39.2 \%)$ & $85,559(42.7 \%)$ \\
\hline Died & $21,639(58.7 \%)$ & $17,819(56.1 \%)$ & $21,321(51.7 \%)$ & $23,022(58.4 \%)$ & $30,990(60.8 \%)$ & $114,791(57.3 \%)$ \\
\hline
\end{tabular}

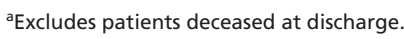


Gupta et al

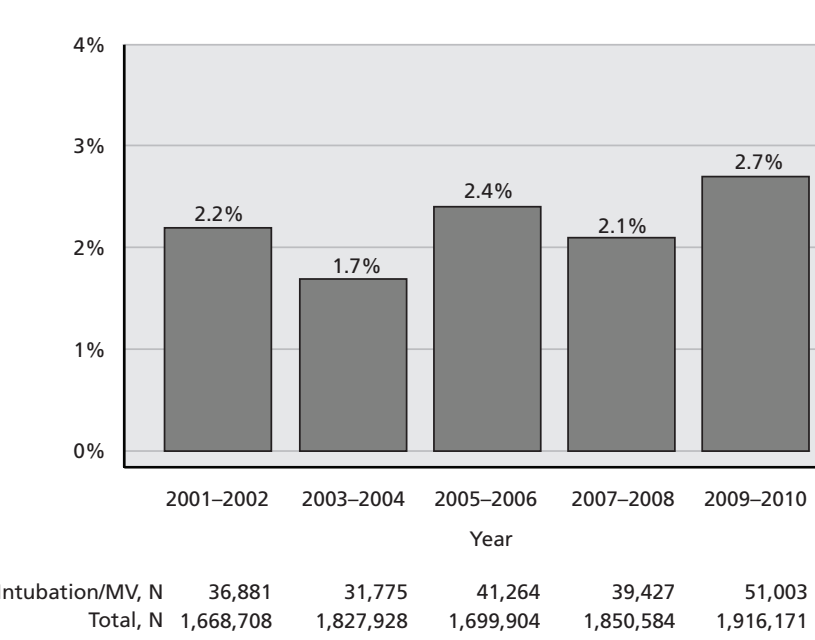

Figure 1. Trends in the percentage and total number of patients with metastatic cancer who underwent intubation and mechanical ventilation (MV) $(P=.032)$.

but their outcomes did not change significantly. More than half of these patients died during hospitalization $(57.3 \%)$, and of those surviving to discharge, approximately $40 \%$ were DTCF. No significant change in outcomes was seen over the study period.

Intubation/MV in patients with cancer is associated with poor outcomes, with studies reporting inpatient mortality rates ranging from $55 \%$ to $83 \% .{ }^{12}$ Outcomes of patients with metastatic cancer admitted to the ICU have reportedly been similar. In our study, we found an overall inpatient mortality of $57.3 \%$, which is similar to previously reported data. ${ }^{13-17}$ However, outcomes may be affected by cancer subtype, comorbidities, severity of acute illness, and the criteria used to admit and discharge patients from the ICU. A recent study evaluated factors associated with 30-day mortality in patients with metastatic cancer requiring intubation/MV and found that nonwhite race, lack of insurance, unscheduled admission, lung cancer as the primary cancer, and comorbidities (eg, liver disease, end-stage renal disease) were associated with poor outcomes. ${ }^{8} \mathrm{~A}$ study in patients with lung cancer found that intubation/MV and severity of organ failure were associated with an increased risk of mortality. ${ }^{18}$ Overall, severity of the acute illness, degree and number of organ dysfunctions, cancer subtype, comorbidities, physical status of patients, and time to ICU admission have been associated with short- and long-term mortality in these patients. ${ }^{19-23}$

Advancements in the treatment of cancer have contributed to patients with metastatic can- cer living longer. Our findings showed that rates of intubation/MV in this population increased only slightly, from $2.2 \%$ to $2.7 \%$. Although this was a statistically significant increase, the absolute change in percentage intubated/MV was small, and the more dramatic increase in total number of patients with metastatic cancer who underwent intubation/MV represents a large increase in eligible patients with metastatic cancer being hospitalized (from 1.67 million admissions in 2001 to 1.92 million in 2010; a $15 \%$ increase). We also observed a similar increase in patients with heart failure and stroke. An increase in the diagnoses of sepsis was also seen through the study period, although data show that changing coding practices are largely responsible for increases in sepsis rates in databases. ${ }^{24}$

Changes in ICU policies, improved ICU management, and early detection of sicker patients have led to overall improved ICU outcomes. ${ }^{4}$ However, we found that improvements in overall cancer and ICU care have not translated into improved outcomes for patients with metastatic cancer requiring MV within the 10-year study period. This may be due to the inherently sicker nature of this population (requiring MV) versus all patients in the ICU. Patients with heart failure and stroke requiring intubation/MV in this study also did not show improved outcomes. Several factors associated with worse outcome, discussed earlier, may have been present in these patients. The increase in palliative care referral over time in patients with metastatic cancer who underwent intubation/MV, as demonstrated in our study, highlights the growing need and subsequent acknowledgement of the importance of palliative care in the management of these patients. The influx of research funding from multiple organizations, recognition of palliative care and hospice care by the American Board of Medical Specialties, and commencement of training of formal palliative care training programs by the Accreditation Council for Graduate Medical Education can partially explain the growing trend of palliative care referral in our study. This trend is also reflected in the National Palliative Care Registry, wherein a similar increment in palliative care has been demonstrated from 2001 to 2010. ${ }^{25}$ Patients who received palliative care in our study had higher mortality, which likely relates to the late referral to palliative care in the ICU and it being reserved for 
A

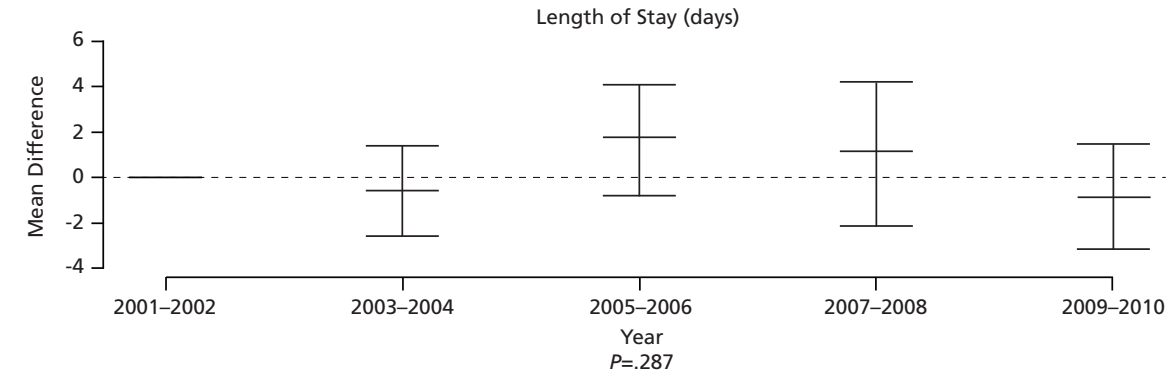

B

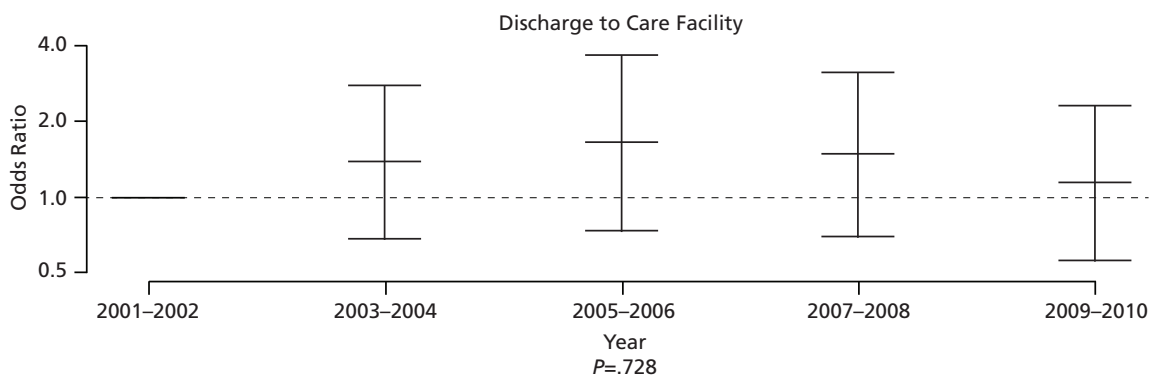

C

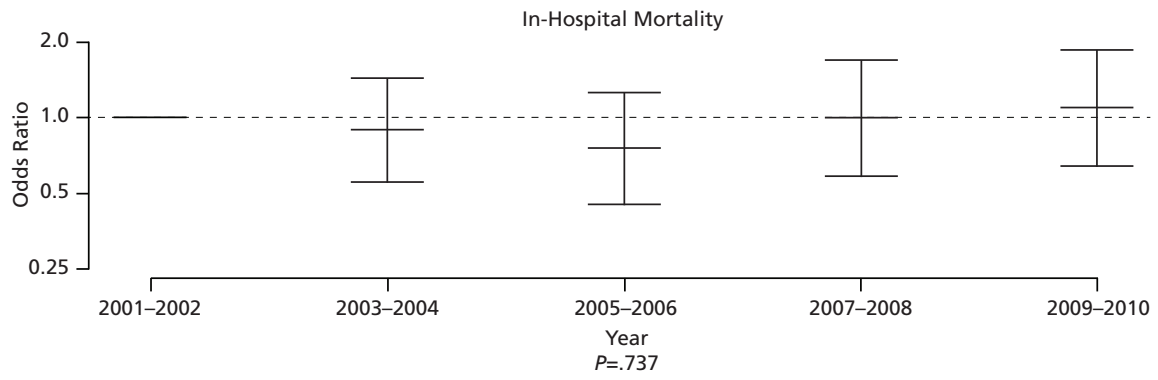

Figure 2. Trends in the outcomes, including (A) length of stay (days), (B) discharge to a care facility, and (C) in-hospital mortality, in patients with metastatic cancer who underwent intubation and mechanical ventilation. Bars represent $95 \%$ confidence intervals.

the sickest patients. Quality improvement projects through the ASCO Quality Training Program have found that early involvement of palliative care in patients with metastatic solid cancer may be associated with reduced ICU admission and mortality. ${ }^{26}$

There are certain limitations to our study. The NHDS database only represents $1 \%$ of patients hospitalized nationally to nonfederal short-stay facilities. Therefore, the results of the study cannot be generalized to all patients with metastatic cancer requiring intubation/MV. We only included patients who required intubation/MV, and therefore our results cannot be generalized to all the patients with cancer requiring ICU admission. Some database-based studies have included patients undergoing arterial line monitoring, central venous pressure monitoring, continuous renal replacement therapy, total parenteral nutrition, and tracheostomy in the ICU; we did not include these patients because some of these interventions can be performed outside the ICU. No information was available regarding the duration of cancer, type of antineoplastic therapy rendered, functional status of the patient, or level or skill of ICU care. We reported inpatient discharge data (including mortality data), but the postdischarge short- and long-term outcomes of these patients remains unknown. Code status was not available in the database, so patients who were designated "do not intubate" may not have been eligible for intubation/MV in the study. The study's retrospective design and lack of information about ICU structure and process are also limitations. Data on healthcare costs were not available, and further details about facilities (rural/urban, teaching/nonteaching) would be useful in addressing the generalizability of these findings. Furthermore, the coding for palliative care is imperfect, with a reported sensitivity and specificity of $81 \%$ to $83 \%$ and $95 \%$ 
to $97 \%$, respectively, although increases in palliative care use has been attributed to changing coding practices. ${ }^{27-29}$

\section{Conclusions}

The outcome of patients with metastatic cancer undergoing intubation/MV has remained poor from
2001 to 2010. Those with metastatic cancer are increasingly eligible for and receiving intubation/MV. Clinicians should discuss potential outcomes with the patient and family as part of the treatment plan and goals of care. Further studies focusing on modifiable risk factors are required for better treatment planning and establishing strategies centered on goals of care for these patients. ${ }^{19-23}$

\section{References}

1. Jemal A, Ward EM, Johnson CJ, et al. Annual report to the nation on the status of cancer, 1975-2014, featuring survival. J Natl Cancer Inst 2017;109:djx030.

2. Puxty K, McLoone P, Quasim T, et al. Risk of critical illness among patients with solid cancers: a population-based observational study. JAMA Oncol 2015;1:1078-1085.

3. McGrath S, Chatterjee F, Whiteley C, Ostermann M. ICU and 6-month outcome of oncology patients in the intensive care unit. QJM 2010;103:397-403.

4. Zimmerman JE, Kramer AA, Knaus WA. Changes in hospital mortality for United States intensive care unit admissions from 1988 to 2012. Crit Care 2013;17:R81.

5. Romano AM, Gade KE, Nielsen G, et al. Early palliative care reduces endof-life intensive care unit (ICU) use but not ICU course in patients with advanced cancer. Oncologist 2017;22:318-323.

6. Ferrell BR, Temel JS, Temin S, et al. Integration of palliative care into standard oncology care: American Society of Clinical Oncology clinical practice guideline update. J Clin Oncol 2017;35:96-112.

7. The Dartmouth Atlas of Health Care. Available at: http://www. dartmouthatlas.org/. Accessed January 21, 2017.

8. Loh KP, Kansagra A, Shieh MS, et al. Predictors of in-hospital mortality in patients with metastatic cancer receiving specific critical care therapies. J Natl Compr Canc Netw 2016;14:979-987.

9. Reisfield GM, Wallace SK, Munsell MF, et al. Survival in cancer patients undergoing in-hospital cardiopulmonary resuscitation: a meta-analysis. Resuscitation 2006;71:152-160.

10. Gupta A, Tariq R, Frank RD, et al. Trends in the incidence and outcomes of hospitalized cancer patients with clostridium difficile infection: a nationwide analysis. J Natl Compr Canc Netw 2017;15:466-472.

11. Sharma RK, Cameron KA, Chmiel JS, et al. Racial/Ethnic differences in inpatient palliative care consultation for patients with advanced cancer. J Clin Oncol 2015;33:3802-3808.

12. Soares M, Depuydt PO, Salluh JI. Mechanical ventilation in cancer patients: clinical characteristics and outcomes. Crit Care Clin 2010;26:4158.

13. Azevedo LC, Caruso P, Silva UV, et al. Outcomes for patients with cancer admitted to the ICU requiring ventilatory support: results from a prospective multicenter study. Chest 2014;146:257-266.

14. Azoulay E, Moreau D, Alberti C, et al. Predictors of short-term mortality in critically ill patients with solid malignancies. Intensive Care Med 2000;26:1817-1823.

15. Caruso P, Ferreira AC, Laurienzo CE, et al. Short- and long-term survival of patients with metastatic solid cancer admitted to the intensive care unit: prognostic factors. Eur J Cancer Care (Engl) 2010;19:260-266.
16. Kress JP, Christenson J, Pohlman AS, et al. Outcomes of critically ill cancer patients in a university hospital setting. Am J Respir Crit Care Med 1999;160:1957-1961.

17. Staudinger T, Stoiser B, Mullner M, et al. Outcome and prognostic factors in critically ill cancer patients admitted to the intensive care unit. Crit Care Med 2000;28:1322-1328.

18. Hwang $\mathrm{KE}$, Seol $\mathrm{CH}$, Hwang YR, et al. The prognosis of patients with lung cancer admitted to the medical intensive care unit. Asia Pac J Clin Oncol 2016;12:e118-124.

19. Loh KP, Kansagra A, Shieh MS, et al. Predictors of the use of specific critical care therapies in patients with metastatic cancer. J Natl Compr Canc Netw 2017;15:22-30.

20. Kostakou E, Rovina N, Kyriakopoulou M, et al. Critically ill cancer patient in intensive care unit: issues that arise. J Crit Care 2014;29:817-822.

21. Schellongowski $P$, Sperr WR, Wohlfarth $P$, et al. Critically ill patients with cancer: chances and limitations of intensive care medicine-a narrative review. ESMO Open 2016;1:e000018.

22. Soares M, Caruso P, Silva E, et al. Characteristics and outcomes of patients with cancer requiring admission to intensive care units: a prospective multicenter study. Crit Care Med 2010;38:9-15.

23. Taccone FS, Artigas AA, Sprung CL, et al. Characteristics and outcomes of cancer patients in European ICUs. Crit Care 2009;13:R15.

24. Rhee C, Murphy MV, Li L, et al. Comparison of trends in sepsis incidence and coding using administrative claims versus objective clinical data. Clin Infect Dis 2015;60:88-95.

25. Rogers M, Dumanovsky T. National Palliative Care Registry Hospital Trends and Insights. Available at: https://registry.capc.org/wp-content/ uploads/2016/09/National-Palliative-Care-Registry_Insights-andTrends-09_15_2016-1.pdf. Accessed January 31, 2018.

26. Cinar P, Bobokalonova Z. ASCO's Quality Training Program: Reducing the Percent of ICU Deaths of Patients With Advanced Cancer at Stanford Health Care. Available at: https://connection.asco.org/sites/ asco_connection/files/Stanford\%20Final\%20Presentation.pdf. Accessed April 16, 2017.

27. Feder S, Tate J, Womack J, et al. Validation of the diagnostic code for palliative care in patients hospitalized with heart failure within the Department of Veterans Affairs (S752). J Pain Symptom Manage 2017;53:438-439.

28. Qureshi AI, Adil MM, Suri MF. Rate of utilization and determinants of withdrawal of care in acute ischemic stroke treated with thrombolytics in USA. Med Care 2013;51:1094-1100.

29. Fekri $O$, Amuah JE, Herasimovich V, et al. Palliative care coding practices in Canada since the introduction of guidelines and the HSMR indicator. BMJ Open 2015;5:e008753.

\section{See JNCCN.org for supplemental online content.}

